\title{
THE DERIVATIVES OF ASIAN CALL OPTION PRICES *
}

\author{
JUNGMIN $\mathrm{CHOI}^{\dagger}$ AND KYOUNGHEE KIM ${ }^{\ddagger}$
}

\begin{abstract}
The distribution of time integrals of geometric Brownian motion is not well understood. To price an Asian option and to obtain measures of its dependence on the parameters of time, strike price, and underlying market price, it is essential to have the distribution of time integral of geometric Brownian motion and it is also required to have a way to manipulate its distribution. We present integral forms for key quantities in the price of Asian option and its derivatives (delta, gamma, theta, and vega $)$. For example for any $a>0, \mathbb{E}\left[\left(A_{t}-a\right)^{+}\right]=t-a+a^{2} \mathbb{E}\left[\left(a+A_{t}\right)^{-1} \exp \left(\frac{2 M_{t}}{a+A_{t}}-\frac{2}{a}\right)\right]$, where $A_{t}=\int_{0}^{t} \exp \left(B_{s}-s / 2\right) d s$ and $M_{t}=\exp \left(B_{t}-t / 2\right)$.
\end{abstract}

Key words. Asian option, derivatives of option prices, geometric Brownian motion, time integral

AMS subject classifications. Primary 91B28, 60J65; Secondary 60G99

\section{Introduction}

The payoff of an Asian option depends on the (geometric or arithmetic) average of prices of a given risky asset over the pre-specified time interval. Under the BlackScholes framework, one assumes that the price process $\left\{S_{t}, t \geq 0\right\}$ of the risky asset follows

$$
d S_{t}=\mu S_{t} d t+\sigma S_{t} d B_{t}, \quad S_{0}>0
$$

where $\mu$ and $\sigma$ are given constants and $\left\{B_{t}, t \geq 0\right\}$ is a standard one dimensional Brownian motion. In this setting, it is easy to understand the geometric average. If $0 \leq t_{1}<t_{2}$ then

$$
\sqrt{S_{t_{1}} \cdot S_{t_{2}}}=S_{0} \exp \left[\left(\sigma \sqrt{t_{2}+3 t_{1}}\right) \cdot \mathcal{N}+\left(\mu-\frac{\sigma^{2}}{2}\right)\left(t_{1}+t_{2}\right)\right]
$$

in distribution, where $\mathcal{N}$ is a standard normal random variable. On the other hand, the distribution of an arithmetic average process is not well understood. A continuous version of the arithmetic average is a time integral of a price process. Using the Inverse Lapalce Transformation, Yor [15] proved many interesting identities related to the distribution of geometric Brownian motion, which gives us deeper understanding of functions of geometric Brownian motion and useful information about their time integrals. More detailed research for the relation between the time integral and an Asian option was considered in [6]. Using the joint density of $\int_{0}^{t} \exp \left(B_{s}\right) d W_{s}, \exp \left(B_{t}\right)$, where $B_{t}, W_{t}$ are independent Brownian motions given in [2], the moment generating function of the time integral process was computed in [11]. The method of changing measures was considered to analyze the properties of the time integral process, (see $[7,12,13,14]$.) In [5] the very useful time reversing property is used to analyze the time integral process. Dufresne also provided a certain form for the density function of the time integral of geometric Brownian motion. However the author pointed out

${ }^{*}$ Received: December 5, 2007; accepted (in revised version): May 1, 2008. Communicated by David Cai.

${ }^{\dagger}$ Mathematics Department Florida State University Tallahassee, FL 32306, USA (choi@math. fsu.edu).

${ }^{\ddagger}$ Mathematics Department Florida State University Tallahassee, FL 32306, USA (kim@math. fsu.edu). 
the difficulties in using his formula in practice, especially when the time integral is over a short time period, due to the slow convergence rate.

The payoff of a European style fixed strike Asian option is given by a function of the time integral of the price of the risky asset $S_{t}$

$$
\left(\frac{1}{|I|} \int_{I} S_{t} d t-\kappa\right)^{+}
$$

where $\kappa$ is a fixed strike price and $I$ is the pre-specified time interval with the length $|I|$. Under the risk neutral measure $\mathbb{Q}$, we may set the price process $S_{t}$ given by a SDE, $d S_{t}=\sigma S_{t} d B_{t}$ where $\sigma$ is a constant depending on the risky asset and $B_{t}$ is a $\mathbb{Q}$-brownian motion. Without loss of generality, we may also assume that the time interval $I=[0, \tau]$ for some $\tau>0$. It follows that the price of a European style Asian option is given by

$$
e^{-r \tau} E_{\mathbb{Q}}\left[\left(\frac{1}{\tau} \int_{0}^{\tau} e^{r t} S_{0} \exp \left\{\sigma B_{t}-\sigma^{2} t / 2\right\} d t-\kappa\right)^{+}\right] .
$$

Since $\sigma B_{t}=B_{\sigma^{2} t}$ in law, we can rewrite the above quantity as follows

$$
\frac{S_{0}}{\tau \sigma^{2}} e^{-r \tau} E_{\mathbb{Q}}\left[\left(\int_{0}^{\sigma^{2} \tau} e^{B_{t}+\left(r / \sigma^{2}-1 / 2\right) t} d t-\frac{\sigma^{2} \kappa \tau}{S_{0}}\right)^{+}\right] .
$$

Note that the initial price of the risky asset is not appeared in the time integral. To obtain the price of Asian option and the derivatives with respect to the asset price we need to understand the following quantities

$$
E\left[\left(A_{t}^{(\mu)}-a\right)^{+}\right], \frac{d}{d a} E\left[\left(A_{t}^{(\mu)}-a\right)^{+}\right], \text {and } \frac{d^{2}}{d a^{2}} E\left[\left(A_{t}^{(\mu)}-a\right)^{+}\right],
$$

where $A_{t}^{(\mu)}=\int_{0}^{t} \exp \left(B_{s}+(\mu-1 / 2) s\right) d s, \mu>0$ and $a>0$. In this paper we show that the quantities in (1.2) can be expressed in terms of the expected values of functions of exponential Brownian motion. We believe that these expressions would provide the alternative approach to simulate the Asian option price and its greeks. The simulation results for Asian option price and its greeks were considered by several authors (for example see $[1,3,4,10]$.

Other types of Asian options are also considered. When the strike price depends on the average price, it is called the floating-strike Asian option (see, for example, [8].) In [8], Henderson and Wojakowski show the very useful symmetries between fixed-strike and floating strike Asian options. They showed that at the starting point of the averaging period there exists an equivalent relation between the floating-strike Asian option and the fixed strike Asian option. However, once the averaging period has begun, the floating strike Asian option can not be re-expressed as a fixed strike option.

In Section 2 we discuss the relation between the time integral and the exponential Brownian motion. We summarize the result in [7] and present the key proposition. In Section 3 we discuss the price of an Asian option and its derivatives (delta, gamma, theta, and vega). In Section 4 we consider the (non-constant) stochastic short rate. Combining our results, we derive an integral form of the call price. This formula is given by the expected value of a differentiable function. 


\section{The time integral of exponential Brownian motion}

For $\mu \in \mathbb{R}_{\geq 0}$ let us denote by $A_{t}^{(\mu)}$ a time integral of an exponential Brownian motion with drift $\mu$

$$
A_{t}^{(\mu)}:=\int_{0}^{t} \exp \left[B_{s}+(\mu-1 / 2) s\right] d s .
$$

When $\mu=0$ we simply use $A_{t}$ without a superscript. For $t \geq 0$ and $y>0$ we set $M_{t}:=\exp \left(B_{t}-t / 2\right)$ and

$$
R_{t}:=-\frac{M_{t}}{y^{-1}-1 / 2 A_{t}}=2 \frac{d}{d t} \log \left(1-\frac{y}{2} A_{t}\right) .
$$

It is not hard to see that the process $R_{t}$ satisfies the following SDE

$$
d R_{t}=R_{t} d B_{t}-\frac{1}{2} R_{t}^{2} d t, \quad R_{0}=-y
$$

up to an explosion time $\tau_{\infty}:=\inf \left\{t \geq 0: A_{t}=2 / y\right\}$. By considering a stopping time $\tau_{n}=\inf \left\{t: R_{t} \leq-n\right\}$ and a Girsanov density process $R_{t} 1_{\left\{t<\tau_{n}\right\}}$, we define a new measure $\mathbb{Q}$ under which

$$
\tilde{B}_{t}=B_{t}-\int_{0}^{t} R_{s} 1_{\left\{s<\tau_{n}\right\}} d s=B_{t}-2 \log \left(1-\frac{y}{2} A_{t \wedge \tau_{n}}\right)
$$

is a standard Brownian motion. Let us define $\tilde{M}=\exp \left(\tilde{B}_{t}-t / 2\right)$ and $\tilde{A}_{t}=\int_{0}^{t} \tilde{M}_{s} d s$. It follows that

$$
\tilde{M}_{t}=\frac{M_{t}}{\left(1-\frac{y}{2} A_{t \wedge \tau_{n}}\right)^{2}}, \quad 1+\frac{y}{2} \tilde{A}_{t \wedge \tau_{n}}=\frac{1}{1-\frac{y}{2} A_{t \wedge \tau_{n}}}
$$

and

$$
R_{t \wedge \tau_{n}}=-\frac{\tilde{M}_{t \wedge \tau_{n}}}{y^{-1}+\frac{1}{2} \tilde{A}_{t \wedge \tau_{n}}}
$$

The following proposition is a simple modification of Proposition 3.3 in [7].

Proposition 2.1. If $f(x, z)$ is a Borel measurable function and $y>0$ then

$$
\begin{aligned}
& E\left[f\left(M_{t}, A_{t}\right) ; A_{t}<\frac{2}{y}\right] \\
& \quad=e^{-y} E\left[f\left(\frac{M_{t}}{\left(1+\frac{y}{2} A_{t}\right)^{2}}, \frac{A_{t}}{1+\frac{y}{2} A_{t}}\right) \exp \left(\frac{M_{t}}{y^{-1}+\frac{1}{2} A_{t}}\right)\right] .
\end{aligned}
$$

Proof. The proof of the proposition is essentially same as the proof of the Proposition 3.3 in [7], so we give a brief sketch (for the detailed proof we refer to [7].) For fixed $n$ and $y>0$ it is not hard to see that

$$
\exp \left(R_{t}+y\right) 1_{\left\{\tau_{n}>t\right\}}=\exp \left(\int_{0}^{t \wedge \tau_{n}} R_{s} d B_{s}-\frac{1}{2} \int_{0}^{t \wedge \tau_{n}} R_{s}^{2} d s\right)
$$

satisfies a Novikov condition. Thus we have

$$
E\left[f\left(M_{t}, A_{t}\right) ; \tau_{n}>t\right]=E_{\mathbb{Q}}\left[f\left(M_{t}, A_{t}\right) \exp \left(-R_{t}-y\right) 1_{\tau_{n}>t}\right] .
$$


Since the event $\left\{\tau_{n}>t\right\}$ is the same as $\left\{\max _{s \leq t} \tilde{M}_{s} /\left(y^{-1}+1 / 2 \tilde{A}_{s}\right)<n\right\}$, by letting $n \rightarrow \infty$ the right hand side becomes $E_{\mathbb{Q}}\left[f\left(M_{t}, A_{t}\right) \exp \left(-R_{t}-y\right)\right]$. Use (2.2) and (2.3) we can rewrite the limit of the right hand side in terms of $\tilde{M}_{t}, \tilde{A}_{t}$ and $\tilde{R}_{t}$. Since we are only interested in the quantity, we remove the tilde and get (2.4).

Let us discuss certain interesting choices of $f$ in Proposition 2.1. Simple choices like a constant function or power functions allows us to have various relations between a simple martingale $M_{t}$ and its time integral. We consider the ones which directly relate to the problem of pricing the European style Asian option and estimating the sensitivities. First we consider a constant function $f(x, z) \equiv 1$. Using a constant function, it is easy to see that the left hand side of (2.4) is a probability distribution of the time integral of a geometric brownian motion. By setting $a=2 / y$ it follows that

Lemma 2.2. For a positive $a>0$, we have

$$
\operatorname{Pr}\left[A_{t}<a\right]=e^{-2 / a} E\left[\exp \left(\frac{M_{t}}{a / 2+1 / 2 A_{t}}\right)\right] .
$$

For $\nu \in \mathbb{R}$ we have $M_{t}^{\nu} \exp \left(\nu t / 2-\nu^{2} t / 2\right)=\exp \left(\nu B_{t}-\nu^{2} t / 2\right)$ and thus

$$
E\left[M_{t}^{\nu} \exp \left(\nu t / 2-\nu^{2} t / 2\right) ; A_{t} \leq 2 / y\right]=\operatorname{Pr}\left[A_{t}^{(\nu)} \leq 2 / y\right] .
$$

By setting $f(x, z)=x^{\nu}$ we get

Lemma 2.3. For a real number $\nu$ and a positive real $a>0$, we have

$$
\begin{aligned}
\operatorname{Pr} & {\left[A_{t}^{(\nu)} \leq a\right] } \\
& =a^{2 \nu} e^{-2 / a} \mathbb{E}\left[\left(a+A_{t}^{(\nu)}\right)^{-2 \nu} \exp \left(\frac{2 \exp \left(B_{t}+(\nu-1 / 2) t\right)}{a+A_{t}^{(\nu)}}\right)\right] .
\end{aligned}
$$

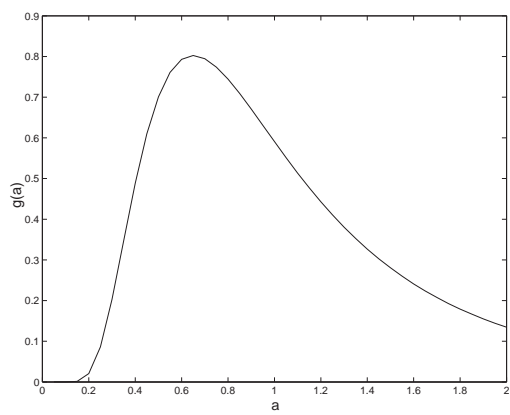

FIG. 2.1. The density function of At.

Let $g_{t}$ denote the probability density function for $A_{t}$. It is known that the density function for $A_{t}$ is continuous and positive. Figure 2.1 is the result of the simulation (10000 simulations for each $a=0.1,0.2, \ldots, 2.0)$ using the identity given in the following Lemma 2.4. The $n$-th moments of the time integral process $A_{t}$ can be computed with 
simple computation. However, it is known that $A_{t}$ has a heavy tail probability and thus knowing all integer moments does not give a probability density function. In Figure 2.1 we can see the heavy tail probability.

Lemma 2.4. For a real number $a>0$, the probability density function $g_{t}$ for $A_{t}$ satisfies

$$
g_{t}(a)=\frac{2}{a^{2}} \operatorname{Pr}\left[A_{t} \leq a\right]-\frac{2}{a^{2}} \operatorname{Pr}\left[A_{t}^{(1)} \leq a\right] .
$$

Furthermore for a real positive number $\nu>0$, the probability density function $g_{t}^{(\nu)}$ for $A_{t}^{(\nu)}$ satisfies

$$
\begin{aligned}
g_{t}^{(\nu)}(a)= & \frac{2}{a^{2}} \operatorname{Pr}\left[A_{t}^{(\nu)} \leq a\right]-\frac{2}{a^{2}} e^{\nu t} \operatorname{Pr}\left[A_{t}^{(\nu+1)} \leq a\right] \\
& +2 \nu a^{2 \nu-1} e^{-2 / a} \mathbb{E}\left[\left(a+A_{t}^{(\nu)}\right)^{-2 \nu-1} \exp \left(\frac{2 \exp \left(B_{t}+(\nu-1 / 2) t\right)}{a+A_{t}^{(\nu)}}\right)\right] .
\end{aligned}
$$

Proof. From Lemma 2.2 we have

$$
\begin{aligned}
g_{t}(a) & =\frac{d}{d a} \operatorname{Pr}\left[A_{t}<a\right] \\
& =\frac{2}{a^{2}} \operatorname{Pr}\left[A_{t} \leq a\right]-\frac{1}{2} e^{-2 / a} E\left[\frac{M_{t}}{\left(a / 2+1 / 2 A_{t}\right)^{2}} \exp \left(\frac{M_{t}}{a / 2+1 / 2 A_{t}}\right)\right] .
\end{aligned}
$$

Since $M_{t}=\exp \left(B_{t}-t / 2\right)$ we use $M_{t}$ as a Girsanov density function for the second term. Under the new measure $B_{t}-t / 2$ is a standard Brownian motion and thus the second term is equal to the following quantity:

$$
\frac{1}{2} e^{-2 / a} E\left[\frac{1}{\left(a / 2+1 / 2 A_{t}^{(1)}\right)^{2}} \exp \left(\frac{2 \exp \left(B_{t}+t / 2\right)}{a+A_{t}^{(1)}}\right)\right] .
$$

Comparing with Lemma 2.3, we can see that the above quantity is the same as $2 / a^{2} \operatorname{Pr}\left[A_{t}^{(1)} \leq a\right]$. For non-zero $\nu$, we do the same computation using Lemma 2.3.

Furthermore we have

Proposition 2.5. For any $a>0, b>0$ and $t>0$ we have

$$
\operatorname{Pr}\left[M_{t}<b, A_{t}<a\right]=e^{-2 / a} \mathbb{E}\left[\exp \left(\frac{2 M_{t}}{a+A_{t}}\right) ; M_{t} \leq b\left(1+\frac{1}{a} A_{t}\right)^{2}\right] .
$$

Proof. Consider $f(x, z)=1\{x<b\}$ in equation (2.4). By setting $a=2 / y$ we get an integration form for the joint probability density function for $M_{t}$ and $A_{t}$.

The simulation result of Proposition 2.5 for $t=a$ is shown on the left of the following figure and the joint distribution for $t=1$ is shown in the right.

The first quantity of interest on the derivatives of Asian options is the expected value of the maximum function of the time integral subtracted by a constant. Using Proposition 2.1 we have the following result:

Theorem 2.6. For any $a>0$ and $\nu \in \mathbf{R}$, we have

$$
\mathbb{E}\left[\left(A_{t}^{(\nu)}-a\right)^{+}\right]=\frac{e^{\nu t}-1}{\nu}-a+e^{\nu t / 2-\nu^{2} t / 2} a^{2 \nu+2} \mathbb{E}\left[\frac{M_{t}^{\nu}}{\left(a+A_{t}\right)^{2 \nu+1}} \exp \left(\frac{2 M_{t}}{a+A_{t}}-\frac{2}{a}\right)\right] .
$$



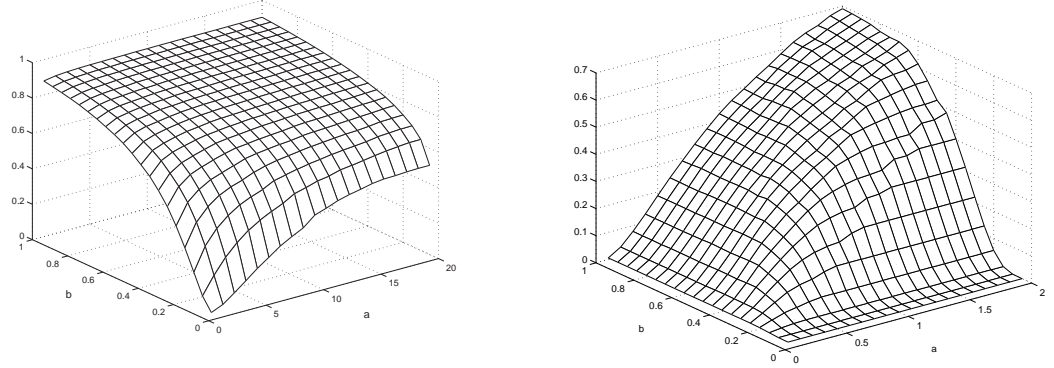

FIG. 2.2. Joint distribution of Mt and At.

In particular, if $\nu=0$ we have

$$
\mathbb{E}\left[\left(A_{t}-a\right)^{+}\right]=t-a+a^{2} \mathbb{E}\left[\left(a+A_{t}\right)^{-1} \exp \left(\frac{2 M_{t}}{a+A_{t}}-\frac{2}{a}\right)\right] .
$$

Proof. Let us set $f(x, z)=x^{\nu}(2 / y-z)$. The left hand side of (2.4) becomes $E\left[2 / y-A_{t} ; A_{t}<2 / y\right]=E\left[2 / y-A_{t}\right]-E\left[\left(A_{t}-2 / y\right)^{+}\right]$. Since $2 / y-A_{t} /\left(1+y / 2 A_{t}\right)=$ $1 /\left(2 / y+A_{t}\right)$, the second result comes directly from (2.4). Using identity (2.5) we can change the drift term of the exponential Brownian motion to get the first result. (

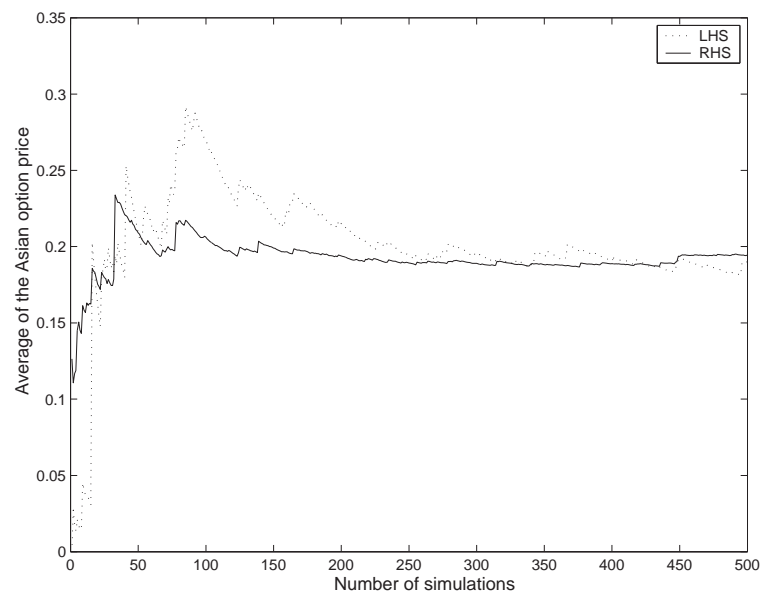

FIG. 2.3. Expected value of max function : $a=0.4, t=0.5$.

The above figure is the simulation result of the second identity (2.7) in Theorem 2.6. We set $a=0.4$ and $t=0.5$. The dotted line is obtained using the left hand side of the equation (2.7), that is, we simulate $A_{t}$ using Monte Carlo methods and take the maximum function. The solid line is from the right hand side of equation (2.7). With only 500 simulations, we can observe that the convergence is faster for the solid line, 
which was expected because of the discarded simulations of the left hand side (when $A_{t}$ is smaller than $a$ ).

Now let us consider the derivatives of $E\left[\left(A_{t}^{(\nu)}-a\right)^{+}\right]$with respect to $a$. Since

$$
E\left[A_{t}^{(\nu)}-a ; A_{t}^{(\nu)} \leq a\right]=-\int_{0}^{a} \operatorname{Pr}\left[A_{t}^{(\nu)} \leq u\right] d u
$$

we have that

$$
\begin{aligned}
E\left[\left(A_{t}-a\right)^{+}\right] & =E\left[A_{t}-a\right]-E\left[A_{t}-a ; ; A_{t} \leq a\right] \\
& =t-a+\int_{0}^{a} \operatorname{Pr}\left[A_{t} \leq u\right] d u
\end{aligned}
$$

and

$$
E\left[\left(A_{t}^{(\nu)}-a\right)^{+}\right]=\frac{1}{\nu}\left(e^{\nu t}-1\right)-a+\int_{0}^{a} \operatorname{Pr}\left[A_{t}^{(\nu)} \leq u\right] d u .
$$

It follows that

$$
\frac{d}{d a} E\left[\left(A_{t}^{(\nu)}-a\right)^{+}\right]=-1+\operatorname{Pr}\left[A_{t}^{(\nu)} \leq a\right], \quad \text { and } \quad \frac{d^{2}}{d a^{2}} E\left[\left(A_{t}^{(\nu)}-a\right)^{+}\right]=g_{t}^{(\nu)}(a) .
$$

The following theorems are the direct applications of Lemma 2.2, Lemma 2.3 and Lemma 2.4.

THEOREM 2.7. The first and second derivatives of $E\left[\left(A_{t}-a\right)^{+}\right]$with respect to a are given by following equations:

$$
\begin{aligned}
\frac{d}{d a} E\left[\left(A_{t}-a\right)^{+}\right]= & -1+e^{-2 / a} \mathbb{E}\left[\exp \left(\frac{2 \exp \left(B_{t}-t / 2\right)}{a+A_{t}}\right)\right] \\
\frac{d^{2}}{d a^{2}} E\left[\left(A_{t}-a\right)^{+}\right]= & \frac{2}{a^{2}} e^{-2 / a} \mathbb{E}\left[\exp \left(\frac{2 \exp \left(B_{t}-t / 2\right)}{a+A_{t}}\right)\right] \\
& -\frac{1}{2} e^{-2 / a} E\left[\frac{M_{t}}{\left(a / 2+1 / 2 A_{t}\right)^{2}} \exp \left(\frac{M_{t}}{a / 2+1 / 2 A_{t}}\right)\right] .
\end{aligned}
$$

THEOREM 2.8. The first and second derivatives of $E\left[\left(A_{t}^{(\nu)}-a\right)^{+}\right]$with respect to a are given by following equations:

$$
\begin{gathered}
\frac{d}{d a} E\left[\left(A_{t}^{(\nu)}-a\right)^{+}\right]=-1+a^{2 \nu} e^{-2 / a} \mathbb{E}\left[\left(a+A_{t}^{(\nu)}\right)^{-2 \nu} \exp \left(\frac{2 \exp \left(B_{t}+(\nu-1 / 2) t\right)}{a+A_{t}^{(\nu)}}\right)\right], \\
\frac{d^{2}}{d a^{2}} E\left[\left(A_{t}^{(\nu)}-a\right)^{+}\right]=\frac{2}{a^{2}} \operatorname{Pr}\left[A_{t}^{(\nu)} \leq a\right]-\frac{2}{a^{2}} e^{\nu t} \operatorname{Pr}\left[A_{t}^{(\nu+1)} \leq a\right] \\
+2 \nu a^{2 \nu-1} e^{-2 / a} \mathbb{E}\left[\left(a+A_{t}^{(\nu)}\right)^{-2 \nu-1} \exp \left(\frac{2 \exp \left(B_{t}+(\nu-1 / 2) t\right)}{a+A_{t}^{(\nu)}}\right)\right] .
\end{gathered}
$$

\section{Sensitivities}

Since $A_{t}$ satisfies $A_{t+s}=A_{t}+M_{t} \tilde{A}_{s}$ for $t, s>0$ where $\tilde{A}$ is an independent copy of $A$, pricing the fixed strike Asian option at time $t>0$ is essentially identical as the pricing the option at the beginning of the averaging period. 
One important activity in Financial Markets is managing risk. One way to measure the risk in the option is estimating the 'Greek letters' such as delta, gamma, theta, etc. In this section we use identities obtained in Section 2 to get the 'Greek letters' of a European Style Asian option under the Black-Scholes framework. The price process of the risky asset follows under the risk-neutral measure

$$
d S_{t}=\sigma S_{t} d B_{t}+r S_{t} d t
$$

where $B_{t}$ is a standard Brownian motion under the risk-neutral measure, $r$ is a constant interest rate and $\sigma$ is a constant volatility of given asset. The expression (1.1) is equal to the price of an European style Asian option with the expiration date $\tau$ and the strike price $\kappa$ :

$$
\text { Call }=\frac{S_{0}}{\tau \sigma^{2}} e^{-r \tau} E\left[\left(\int_{0}^{\sigma^{2} \tau} e^{B_{t}+\left(r / \sigma^{2}-1 / 2\right) t} d t-\frac{\sigma^{2} \kappa \tau}{S_{0}}\right)^{+}\right] .
$$

THEOREM 3.1. The price of an Asian call at time $t=0$ is given by

$$
\begin{aligned}
\text { Call }_{0} & =\frac{S_{0}}{\tau} e^{-r \tau}\left[\frac{e^{r \tau}-1}{r}-a / \sigma^{2}+\right. \\
& \left.+\frac{e^{\left(r-r^{2} / \sigma^{2}\right) \tau / 2} a^{2 r / \sigma^{2}+2}}{\sigma^{2}} \mathbb{E}\left[\frac{M_{\sigma^{2} \tau}^{r / \sigma^{2}}}{\left(a+A_{\sigma^{2} \tau}\right)^{2 r / \sigma^{2}+1}} \exp \left(\frac{2 M_{\sigma^{2} \tau}}{a+A_{\sigma^{2} \tau}}-\frac{2}{a}\right)\right]\right],
\end{aligned}
$$

where $a=\frac{\sigma^{2} \kappa \tau}{S_{0}}, \tau$ is the expiration date, $\kappa$ is the strike price.

Proof. The result comes directly from the combination of Theorem 2.6 and Equ. (3.1).

The delta $\Delta$ is the rate of change of the price of the option with respect to the price of the underline asset. Thus we have

$$
\Delta=\frac{C a l l_{0}}{S_{0}}-\left.\frac{\kappa}{S_{0}} e^{-r \tau} \frac{d}{d a} E\left[\left(A_{\sigma^{2} \tau}^{(r)}-a\right)^{+}\right]\right|_{a=\frac{\sigma^{2} \kappa \tau}{S_{0}}} .
$$

Using Theorem 2.6 and Theorem 2.7 we have

TheOrem 3.2. The delta $\Delta_{0}$ of an Asian call option at time $t=0$ is given by

$$
\Delta_{0}=\frac{\operatorname{Call}_{0}}{S_{0}}+\frac{\kappa}{S_{0}} e^{-r \tau}\left(1-\operatorname{Pr}\left[A_{\sigma^{2} \tau}^{(r)} \leq \frac{\sigma^{2} \kappa \tau}{S_{0}}\right]\right) .
$$

The gamma $\Gamma$ is the rate of change of the delta with respect to the price of underlying asset. Thus the direct computation using Equ. (3.2) and the results in Theorem 2.6, Theorem 2.7 and Theorem 3.2 we have

TheOrem 3.3. The gamma $\Gamma_{0}$ of an Asian call option at time $t=0$ is given by

$$
\Gamma_{0}=\frac{\sigma^{2} \kappa^{2} \tau}{S_{0}^{3}} e^{-r \tau} g_{\tau \sigma^{2}}^{(r)}\left(\frac{\sigma^{2} \kappa \tau}{S_{0}}\right)
$$

where $g_{t}^{(r)}$ is a continuous probability density function of $A_{t}^{(r)}$ given in Lemma 2.4. 

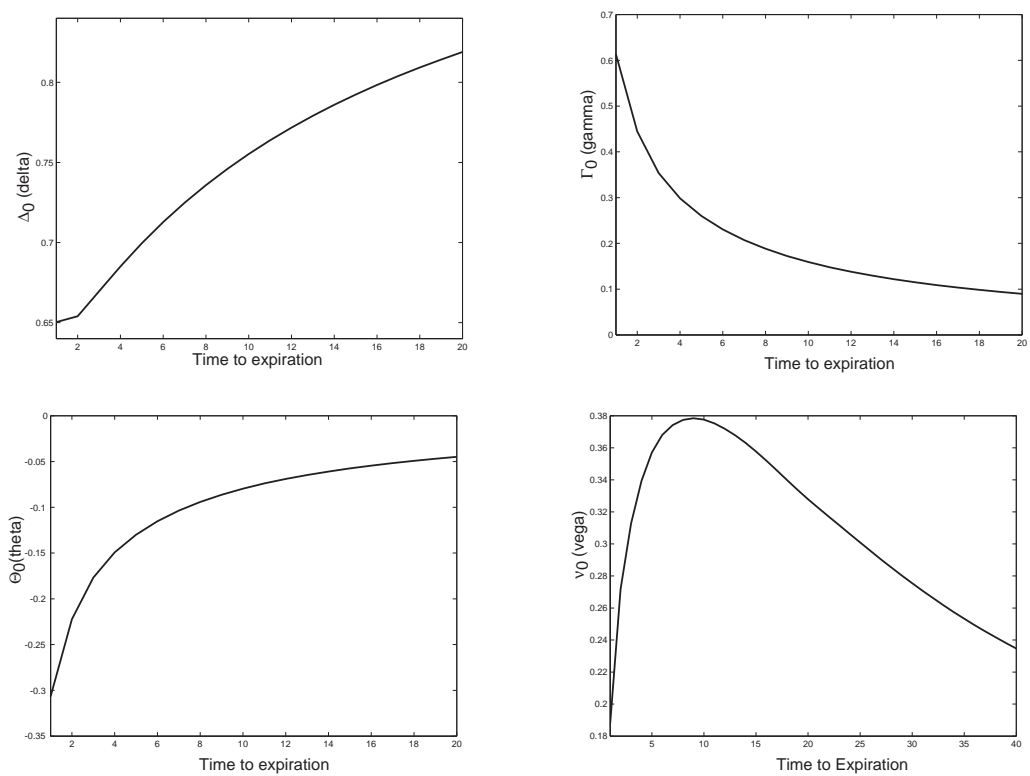

FIG. 3.1. Derivatives vs. the time to expiration: $S 0=\kappa=\sigma=1$.

In Figure 3.1. we use Monte Carlo methods to get Greek letters $\Delta, \Gamma, \Theta$ and $\nu$. We take the risk free rate $r=0$ and both the initial stock price and the strike price are equal to 1 . We also set for simplicity $\sigma=1$. Notice that we plot greek letters vs. time to expiration. Since we computed greeks at the beginning of the averaging period, time to expiration is the same as the length of the averaging period. In the upper left. figure we can observe that as time to expiration increases, the value of $\Delta_{0}$ increases. The plot of $\Gamma_{0}$ with respect to time to expiration is given in the upper right. figure. Since $\Gamma$ is the first derivative of $\Delta$ with respect to the stock prices, it reflects our observation in the plot of $\Delta_{0}$. The lower left figure is the plot of $\Theta_{0}$ and the lower right figure is the plot of $\nu_{0}$.

Since all parameters are constants, the call price satisfies the differential equation

$$
\frac{\partial \text { Call }}{\partial t}+r S \frac{\partial C a l l}{\partial S}+\frac{1}{2} \sigma^{2} S \frac{\partial^{2} \text { Call }}{\partial S^{2}}=r \text { Call } .
$$

It follows that

$$
\Theta_{0}+r S_{0} \Delta_{0}+\frac{1}{2} \sigma^{2} S_{0} \Gamma_{0}=r \text { Call }_{0}
$$

where $\Theta_{0}$ is the rate of change of the price of the option at time $t=0$ with respect to time and $\mathrm{Call}_{0}$ is the price of the call option at time 0 .

Theorem 3.4. The theta $\Theta_{0}$ of an Asian call option at time $t=0$ is given by

$$
\Theta_{0}=r C a l l_{0}-r S_{0} \Delta_{0}-\frac{1}{2} \sigma^{2} S_{0} \Gamma_{0}
$$

where Call $_{0}, \Delta_{0}$ and $\Gamma_{0}$ are given in the previous Theorems 3.1-3. 
Now let us discuss the vega $\nu$. The vega $\nu$ is the rate of change of the price of the option with respect to the volatility of the underlying asset. The call price of European style Asian option is given in (3.1). Thus we have

$$
\begin{aligned}
\frac{\partial C a l l}{\partial \sigma}=-\frac{2}{\sigma} \text { Call }+\frac{2 S_{0}}{\sigma} e^{-r \tau+r \sigma^{2} \tau / 2} E\left[M_{\sigma^{2} \tau}-\frac{\kappa}{S_{0}} ; A_{\sigma^{2} \tau}^{(r)}>\frac{\sigma^{2} \kappa \tau}{S_{0}}\right] \\
=-\frac{2}{\sigma} \text { Call }+\frac{2 S_{0}}{\sigma} e^{-r \tau+r \sigma^{2} \tau / 2} E\left[M_{\sigma^{2} \tau}-\frac{\kappa}{S_{0}}\right] \\
\\
-\frac{2 S_{0}}{\sigma} e^{-r \tau+r \sigma^{2} \tau / 2} E\left[M_{\sigma^{2} \tau}-\frac{\kappa}{S_{0}} ; A_{\sigma^{2} \tau}^{(r)}<\frac{\sigma^{2} \kappa \tau}{S_{0}}\right] .
\end{aligned}
$$

It follows that

$$
\begin{gathered}
\frac{\partial \text { Call }}{\partial \sigma}=-\frac{2}{\sigma} \text { Call }+\frac{2 S_{0}}{\sigma} e^{-r \tau+r \sigma^{2} \tau / 2}\left(1-E\left[M_{\sigma^{2} \tau} ; A_{\sigma^{2} \tau}^{(r)}<\frac{\sigma^{2} \kappa \tau}{S_{0}}\right]\right) \\
-\frac{2 \kappa}{\sigma} e^{-r \tau+r \sigma^{2} \tau / 2}\left(1-P\left[A_{\sigma^{2} \tau}^{(r)}<\frac{\sigma^{2} \kappa \tau}{S_{0}}\right]\right) .
\end{gathered}
$$

By setting $\nu=1$ in Equ. (2.5) in Lemma 2.2, we see that

$$
1-E\left[M_{\sigma^{2} \tau} ; A_{\sigma^{2} \tau}^{(r)}<\frac{\sigma^{2} \kappa \tau}{S_{0}}\right]=P\left[A_{\sigma^{2} \tau}^{(r+1)}>\frac{\sigma^{2} \kappa \tau}{S_{0}}\right] .
$$

Therefore we have

THEOREM 3.5. The vega $\nu_{0}$ of an asian call option at time $t=0$ is given by

$$
\begin{aligned}
\nu_{0}=-\frac{2}{\sigma} C_{a l l_{0}}+ & \frac{2 S_{0}}{\sigma} e^{-r \tau+r \sigma^{2} \tau / 2} P\left[A_{\sigma^{2} \tau}^{(r+1)}>\frac{\sigma^{2} \kappa \tau}{S_{0}}\right] \\
& -\frac{2 \kappa}{\sigma} e^{-r \tau+r \sigma^{2} \tau / 2} P\left[A_{\sigma^{2} \tau}^{(r)}>\frac{\sigma^{2} \kappa \tau}{S_{0}}\right] .
\end{aligned}
$$

Last two quantities in the above theorem can be obtained using Lemma 2.3.

\section{Non-constant interest rates}

In this section we extend our method to the stochastic instantaneous risk-free interest rate $r(t)$. In future work we will consider stochastic interest rates and (or) stochastic volatility. At present we show that the call price of the European style Asian option can be written as the integral of a differentiable function of parameters. The money market account $g_{T}$ at time $T \geq 0$ is given by

$$
g_{T}=e^{\int_{0}^{T} r(t) d t} .
$$

Thus the price of the European style Asian Call option at time $t=0$ is following:

$$
\begin{aligned}
\operatorname{Call}_{0} & =\mathbb{E}_{\mathbb{Q}}\left[e^{-\int_{0}^{\tau} r(t) d t}\left(\frac{1}{\tau} \int_{0}^{\tau} S_{t} d t-\kappa\right)^{+}\right] \\
& =\mathbb{E}_{\mathbb{Q}}\left[e^{-\int_{0}^{\tau} r(t) d t}\left(\frac{1}{\tau} \int_{0}^{\tau} S_{0} e^{\sigma B_{t}-\sigma^{2} t / 2+\int_{0}^{t} r(s) d s} d t-\kappa\right)^{+}\right] .
\end{aligned}
$$


Let us consider a discrete model for the short rate

$$
\begin{aligned}
& r_{n+1}-r_{n}=\theta\left(r_{n}\right) \Delta t+\rho\left(r_{n}\right) \Delta W_{n}, \\
& r(t)=r_{n} \quad \text { for } t \in\left[t_{n}, t_{n+1}\right)
\end{aligned}
$$

where $W_{t}$ is a standard Brownian motion (not necessarily independent to $B_{t}$ ), $t_{0}=0$, $r_{n}=r\left(t_{n}\right), t_{n}=n \Delta t, \Delta t=t_{n+1}-t_{n}, \Delta W_{n}=W_{t_{n+1}}-W_{t_{n}}$ for $n=0,1,2, \ldots$ and $\theta$ and $\rho$ are real valued functions. From the fact that Brownian motion has independent increments, for some $0<T<\tau$ we have

$$
\begin{aligned}
\int_{0}^{\tau} e^{\sigma B_{t}-\sigma^{2} t / 2+\int_{0}^{t} r(s) d s} d t & =\int_{0}^{T} e^{\sigma B_{t}-\sigma^{2} t / 2+\int_{0}^{t} r(s) d s} d t \\
& +e^{\sigma B_{T}-\sigma^{2} T / 2+\int_{0}^{T} r(s) d s} \int_{0}^{\tau-T} e^{\sigma \tilde{B}_{t}-\sigma^{2} t / 2+\int_{0}^{t} r(s+T) d s} d t
\end{aligned}
$$

where $\tilde{B}_{t}$ is an independent Brownian motion to $B_{t}$. Let us set

$$
m:=\max \left\{n: t_{n}<\tau\right\} .
$$

It follows that using the above Equ. (4.2) we have

$$
\begin{aligned}
\text { Call }_{0} & =\mathbb{E}_{\mathbb{Q}}\left[\mathbb{E}_{\mathbb{Q}}\left[e^{-\int_{0}^{\tau} r(t) d t}\left(\frac{1}{\tau} \int_{0}^{\tau} S_{0} e^{\sigma B_{t}-\sigma^{2} t / 2+\int_{0}^{t} r(s) d s} d t-\kappa\right)^{+} \mid \mathcal{F}_{t_{m}}\right]\right] \\
& =\mathbb{E}_{\mathbb{Q}}\left[\mathbb{E}_{\mathbb{Q}}\left[\mathcal{C}_{0}(t)\left(\mathcal{C}_{1}(t) \int_{0}^{\tau-t_{m}} e^{\sigma \tilde{B}_{t}-\sigma^{2} t / 2+r_{m} t} d t-\mathcal{C}_{2}(t)\right)^{+} \mid \mathcal{F}_{t_{m}}\right]\right]
\end{aligned}
$$

where

$$
\begin{aligned}
& \mathcal{C}_{0}(t)=e^{-\int_{0}^{t_{m}} r(t) d t} e^{r_{m}\left(\tau-t_{m}\right)} S_{0} / \tau \\
& \mathcal{C}_{1}(t)=e^{\sigma B_{t_{m}}-\sigma^{2} t_{m} / 2+\int_{0}^{t_{m}} r(s) d s} \\
& \mathcal{C}_{2}(t)=\kappa \tau / S_{0}-\int_{0}^{t_{m}} e^{\sigma B_{t}-\sigma^{2} t / 2+\int_{0}^{t} r(s) d s} d t
\end{aligned}
$$

and $r_{m}, \mathcal{C}_{j}(t), j=0,1,2$ are measurable in $\mathcal{F}_{t m}$. Thus we have

$$
\operatorname{Call}_{0}=\mathbb{E}_{\mathbb{Q}}\left[\mathcal{C}_{0}(t) \mathcal{C}_{1}(t) \mathbb{E}_{\mathbb{Q}}\left[\left(\int_{0}^{\tau-t_{m}} e^{\sigma \tilde{B}_{t}-\sigma^{2} t / 2+r_{m} t} d t-\frac{\mathcal{C}_{2}(t)}{\mathcal{C}_{1}(t)}\right)^{+} \mid \mathcal{F}_{t_{m}}\right]\right]
$$

Using Theorem 2.6 the inner integral can be rewritten as:

$$
\phi_{1}\left(r_{m}\right)+\phi\left(r_{m}\right) \mathbb{E}\left[\frac{\tilde{M}_{\sigma^{2}\left(\tau-t_{m}\right)}^{r_{m} / \sigma^{2}}}{\left(a+\tilde{A}_{\sigma^{2}\left(\tau-t_{m}\right)}\right)^{2 r_{m} / \sigma^{2}+1}} \exp \left(\frac{2 \tilde{M}_{\sigma^{2}\left(\tau-t_{m}\right)}}{\left.a+\tilde{A}_{\sigma^{2}\left(\tau-t_{m}\right.}\right)}-\frac{2}{a}\right)\right] .
$$

where $a=\sigma^{2} \mathcal{C}_{2}(t) / \mathcal{C}_{1}(t)$, and

$$
\begin{aligned}
& \tilde{M}_{t}=\exp \left(\tilde{B}_{t}-t / 2\right), \quad \tilde{A}_{t}^{(\nu)}=\int_{0}^{t} \exp \left(\tilde{B}_{t}+(\nu-1 / 2) t\right) \\
& \phi_{1}\left(r_{m}, t\right)=\left(e^{r_{m}\left(\tau-t_{m}\right)}-1\right) / r_{m}-a / \sigma^{2} \\
& \phi_{2}\left(r_{m}, t\right)=e^{r_{m}\left(\tau-t_{m}\right) / 2-r_{m}^{2}\left(\tau-t_{m}\right) /\left(2 \sigma^{2}\right)} a^{2 r_{m} / \sigma^{2}+2} / \sigma^{2} .
\end{aligned}
$$


It follows that the call price is given by the following expectation of a differentiable function of parameters such as $S_{0}, \sigma, t$ :

TheOREM 4.1. The price of an Asian call at time $t=0$ is given by

$$
\text { Call }_{0}=\mathbb{E}\left[\mathcal{C}_{0}(t) \mathcal{C}_{1}(t)\left(\phi_{1}\left(r_{m}\right)+\phi\left(r_{m}\right) \mathcal{M}\right)\right],
$$

where $\tau$ is the expiration date, $\kappa$ is the strike price, $\mathcal{C}_{j}(t), j=0,1,2$ are given in (4.3), $\phi_{j}, j=1,2$ are given in (4.5) and

$$
\mathcal{M}=\mathbb{E}\left[\frac{\tilde{M}_{\sigma^{2}\left(\tau-t_{m}\right)}^{r_{m} / \sigma^{2}}}{\left(a+\tilde{A}_{\sigma^{2}\left(\tau-t_{m}\right)}\right)^{2 r_{m} / \sigma^{2}+1}} \exp \left(\frac{2 \tilde{M}_{\sigma^{2}\left(\tau-t_{m}\right)}}{a+\tilde{A}_{\sigma^{2}\left(\tau-t_{m}\right)}}-\frac{2}{a}\right)\right] .
$$

Acknowledgement. We wish to thank the referee for helpful comments on this paper, and Kiseop Lee for finding an error in an earlier version.

\section{REFERENCES}

[1] J. Andreasen, The pricing of discretely sampled Asian and lookback options: a change of numeraire approach, J. Comp. Finance, 1, 15-36, 1998.

[2] P. Bougerol, Examples de théprèmes locaux sur les groups résolubles, Ann. Inst. H. Poincaré Sect. B (N.S.), 19, 369-391, 1983.

[3] M. Broadie and P. Glasserman, Estimating security price derivatives using simulation, Management Science, 42, 269-285, 1996.

[4] S.L. Chung, M. Shackleton and R. Wojakowski, Efficient quadratic approximation of floating strike Asian option values, Finance, 24, 49-62, 2003.

[5] D. Dufresne, The integral of geometric Brownian motion, Adv. in Appl. Probab., 33, 223-241, 2001.

[6] H. Geman and M. Yor, Asian options, Bessel processes and perpetuities, Math. Finance, 2, 349-375, 1993.

[7] V. Goodman and K. Kim: Exponential martingales and time integrals of Brownian motion, preprint, 2006, math.PR/0612034.

[8] V. Henderson and R. Wojakowski, On the equivalence of floating and fixed-strike asian options, J. Appl. Prob., 39, 391-394, 2002.

[9] J.C. Hull, Options, Futures, and Other Derivatives, 6th Edition, Prentice Hall, 2006.

[10] N. Ju, Pricing Asian and basket options via Taylor expansion, J. Comp. Finance, 5, 79-103, 2002.

[11] K. Kim, Moment generating function of the inverse of integral of geometric Brownian motion, Proc. Amer. Math. Soc., 132, 2753-2759, 2004.

[12] H. Matsumoto and M. Yor, An analogue of Pitman's 2M - X theorem for exponential Wiener functionals, part I: a time-inversion approach, Nagoya Math. J., 159, 125-166, 2000.

[13] H. Matsumoto and M. Yor, An analogue of Pitman's $2 M-X$ theorem for exponential Wiener functionals, part II: the role of the generalized inverse Gaussian laws, Nagoya Math. J., 162, 65-86, 2001.

[14] H. Matsumoto and M. Yor, A relationship between Brownian motions with opposite drifts via certain enlargments of the Brownian filtration, Osaka J. Math. 38, 383-398 2001.

[15] M. Yor, On some exponential functionals of Brownian motion, Adv. in Appl. Probab., 24, 509-531, 1992. 